\title{
IMPROVING SCHWARZ INEQUALITY IN INNER PRODUCT SPACES
}

\author{
SILVESTRU SEVER DRAGOMIR ${ }^{1,2}$
}

\begin{abstract}
Some improvements of the celebrated Schwarz inequality in complex inner product spaces are given. Applications for $n$-tuples of complex numbers are provided.
\end{abstract}

\section{INTRODUCTION}

Let $(H,\langle\cdot, \cdot\rangle)$ be an inner product space over the real or complex numbers field $\mathbb{K}$. The following inequality is well known in literature as the Schwarz inequality

$$
\|x\|^{2}\|y\|^{2} \geq|\langle x, y\rangle|^{2}
$$

for any $x, y \in H$. The equality case holds in (1.1) if and only if there exists a constant $\lambda \in \mathbb{K}$ such that $x=\lambda y$. This inequality can be written in an equivalent form as

$$
\|x\|\|y\| \geq|\langle x, y\rangle|
$$

Assume that $P: H \rightarrow H$ is an orthogonal projection on $H$, namely, it satisfies the condition $P^{2}=P=P^{*}$. We obviously have in the operator order of $B(H)$, the Banach algebra of all linear bounded operators on $H$, that $0 \leq P \leq 1_{H}$.

In the recent paper [5. Eq. (2.6)] we established among others that

$$
\|x\|\|y\| \geq\langle P x, x\rangle^{1 / 2}\langle P y, y\rangle^{1 / 2}+|\langle x, y\rangle-\langle P x, y\rangle|
$$

for any $x, y \in H$. Since by the triangle inequality we have

$$
|\langle x, y\rangle-\langle P x, y\rangle| \geq|\langle x, y\rangle|-|\langle P x, y\rangle|
$$

and by the Schwarz inequality for nonnegative selfadjoint operators we have

$$
\langle P x, x\rangle^{1 / 2}\langle P y, y\rangle^{1 / 2} \geq|\langle P x, y\rangle|
$$

for any $x, y \in H$, then we get from (1.3) the following refinement of (1.2)

$$
\|x\|\|y\|-|\langle x, y\rangle| \geq\langle P x, x\rangle^{1 / 2}\langle P y, y\rangle^{1 / 2}-|\langle P x, y\rangle| \geq 0
$$

for any $x, y \in H$.

In 1985 the author [1] (see also 2] or [4, p. 36]) established the following inequality related to Schwarz inequality

$$
\left(\|x\|^{2}\|z\|^{2}-|\langle x, z\rangle|^{2}\right)\left(\|y\|^{2}\|z\|^{2}-|\langle y, z\rangle|^{2}\right) \geq\left|\langle x, y\rangle\|z\|^{2}-\langle x, z\rangle\langle z, y\rangle\right|^{2}
$$

1991 Mathematics Subject Classification. 46C05; 26D15.

Key words and phrases. Inner product spaces, Schwarz's inequality. 
for any $x, y, z \in H$ and obtained, as a consequence, the following refinement of (1.2):

$$
\|x\|\|y\| \geq|\langle x, y\rangle-\langle x, e\rangle\langle e, y\rangle|+|\langle x, e\rangle\langle e, y\rangle| \geq|\langle x, y\rangle|
$$

for any $x, y, e \in H$ with $\|e\|=1$.

If we take the square root in (1.5) and use the triangle inequality, we get for $x$, $y, z \in H \backslash\{0\}$ that

$$
\begin{aligned}
& \left(\|x\|^{2}\|z\|^{2}-|\langle x, z\rangle|^{2}\right)^{1 / 2}\left(\|y\|^{2}\|z\|^{2}-|\langle y, z\rangle|^{2}\right)^{1 / 2} \\
& \geq\left|\langle x, y\rangle\|z\|^{2}-\langle x, z\rangle\langle z, y\rangle\right| \geq|\langle x, z\rangle\langle z, y\rangle|-|\langle x, y\rangle|\|z\|^{2}
\end{aligned}
$$

which by division with $\|x\|^{2}\|y\|^{2}\|z\|^{2} \neq 0$ produces

$$
\frac{|\langle x, y\rangle|}{\|x\|\|y\|} \geq \frac{|\langle x, z\rangle|}{\|x\|\|z\|} \frac{|\langle z, y\rangle|}{\|z\|\|y\|}-\sqrt{1-\frac{|\langle x, z\rangle|^{2}}{\|x\|^{2}\|z\|^{2}}} \sqrt{1-\frac{|\langle y, z\rangle|^{2}}{\|y\|^{2}\|z\|^{2}}} .
$$

If the angle between the vectors $x, y, \Psi_{x y} \in[0, \pi / 2]$, is defined by 8 .

$$
\cos \Psi_{x y}=\frac{|\langle x, y\rangle|}{\|x\|\|y\|}, x, y \neq 0
$$

then the function $\Psi_{x y}$ is a natural metric on complex projective space, since is satisfies the inequality [8]

$$
\Psi_{x y} \leq \Psi_{x z}+\Psi_{z y} \text { for any } x, y, z \neq 0 .
$$

By using (1.8) we have by (1.7) that

$$
\cos \Psi_{x y} \geq \cos \Psi_{x z} \cos \Psi_{z x}-\sin \Psi_{x z} \sin \Psi_{z x}=\cos \left(\Psi_{x z}+\Psi_{z x}\right),
$$

which is equivalent to (1.9) since the function $\cos$ is decreasing on $[0, \pi]$. This provides a different proof of (1.9) than the one from [8] where it was done by utilising the celebrated Krĕ̌n's inequality [7, see also [6. p. 56],

$$
\Phi_{x, y} \leq \Phi_{x, z}+\Phi_{x, z} \text { for any } x, y, z \neq 0,
$$

obtained for angles $\Phi_{x, y}$ between two vectors $x, y$, where in this case $\Phi_{x, y}$ is defined by

$$
\cos \Phi_{x, y}=\frac{\operatorname{Re}\langle x, y\rangle}{\|x\|\|y\|}, x, y \neq 0 .
$$

The following inequality has been obtained by Wang and Zhang in [10] (see also [11, p. 195])

$$
\sqrt{1-\frac{|\langle x, y\rangle|^{2}}{\|x\|^{2}\|y\|^{2}}} \leq \sqrt{1-\frac{|\langle x, z\rangle|^{2}}{\|x\|^{2}\|z\|^{2}}}+\sqrt{1-\frac{|\langle y, z\rangle|^{2}}{\|y\|^{2}\|z\|^{2}}}
$$

for any $x, y, z \in H \backslash\{0\}$. Using the above notations the inequality (1.11) can be written as 8 ]

$$
\sin \Psi_{x, y} \leq \sin \Psi_{x, z}+\sin \Psi_{x, z}
$$

for any $x, y, z \in H \backslash\{0\}$. It also provides another triangle type inequality complementing the KreIn and Lin inequalities above.

The corresponding result for the angle $\Phi_{x, y}$ was obtained by Lin in $[8$ as

$$
\sin \Phi_{x, y} \leq \sin \Phi_{x, z}+\sin \Phi_{x, z}, \text { for any } x, y, z \neq 0,
$$


or, equivalently, as

$$
\sqrt{1-\frac{|\operatorname{Re}\langle x, y\rangle|^{2}}{\|x\|^{2}\|y\|^{2}}} \leq \sqrt{1-\frac{|\operatorname{Re}\langle x, z\rangle|^{2}}{\|x\|^{2}\|z\|^{2}}}+\sqrt{1-\frac{|\operatorname{Re}\langle y, z\rangle|^{2}}{\|y\|^{2}\|z\|^{2}}}
$$

for any $x, y, z \neq 0$.

In [8] the author has also shown that, in fact, the inequalities (1.11) and (1.14) can be extended for any power $p>2$, namely as

$$
\left(1-\frac{|\langle x, y\rangle|^{p}}{\|x\|^{p}\|y\|^{p}}\right)^{1 / p} \leq\left(1-\frac{|\langle x, z\rangle|^{p}}{\|x\|^{p}\|z\|^{p}}\right)^{1 / p}+\left(1-\frac{|\langle y, z\rangle|^{p}}{\|y\|^{p}\|z\|^{p}}\right)^{1 / p}
$$

and

$$
\left(1-\frac{|\operatorname{Re}\langle x, y\rangle|^{p}}{\|x\|^{p}\|y\|^{p}}\right)^{1 / p} \leq\left(1-\frac{|\operatorname{Re}\langle x, z\rangle|^{p}}{\|x\|^{p}\|z\|^{p}}\right)^{1 / p}+\left(1-\frac{|\operatorname{Re}\langle y, z\rangle|^{p}}{\|y\|^{p}\|z\|^{p}}\right)^{1 / p}
$$

for any $x, y, z \neq 0$.

In this paper we obtain some improvements of Schwarz inequality in complex inner product spaces as follows. For various inequalities related to this famous result see the monographs 3 , and [4].

\section{Main Results}

Employing Lin's inequalities (1.15) and (1.16) we can obtain the following refinements of Schwarz's inequality.

Theorem 1. Let $x, y, e \in H$ with $\|e\|=1$ and $p \geq 2$. Then we have the following refinements of Schwarz inequality

$$
\|x\|^{p}\|y\|^{p}-|\langle x, y\rangle|^{p} \geq\left(\operatorname{det}\left[\begin{array}{cc}
\|x\| & \left(\|x\|^{p}-|\langle x, e\rangle|^{p}\right)^{1 / p} \\
\|y\| & \left(\|y\|^{p}-|\langle y, e\rangle|^{p}\right)^{1 / p}
\end{array}\right]\right)^{p}
$$

and

$$
\|x\|^{p}\|y\|^{p}-|\operatorname{Re}\langle x, y\rangle|^{p} \geq\left(\operatorname{det}\left[\begin{array}{cc}
\|x\| & \left(\|x\|^{p}-|\operatorname{Re}\langle x, e\rangle|^{p}\right)^{1 / p} \\
\|y\| & \left(\|y\|^{p}-|\operatorname{Re}\langle y, e\rangle|^{p}\right)^{1 / p}
\end{array}\right]\right)^{p} .
$$

Proof. We observe that, by (1.15) and (1.16)

$$
d_{p}(x, y):=\left(1-\frac{|\langle x, y\rangle|^{p}}{\|x\|^{p}\|y\|^{p}}\right)^{1 / p} \text { and } \delta_{p}(x, y):=\left(1-\frac{|\operatorname{Re}\langle x, y\rangle|^{p}}{\|x\|^{p}\|y\|^{p}}\right)^{1 / p}
$$

are distances and by the continuity property of the distance $d$, namely

$$
|d(x, z)-d(y, z)| \leq d(x, y)
$$

we get

$$
\left|\left(1-\frac{|\langle x, e\rangle|^{p}}{\|x\|^{p}}\right)^{1 / p}-\left(1-\frac{|\langle y, e\rangle|^{p}}{\|y\|^{p}}\right)^{1 / p}\right| \leq\left(1-\frac{|\langle x, y\rangle|^{p}}{\|x\|^{p}\|y\|^{p}}\right)^{1 / p}
$$

and

$$
\left|\left(1-\frac{|\operatorname{Re}\langle x, e\rangle|^{p}}{\|x\|^{p}}\right)^{1 / p}-\left(1-\frac{|\operatorname{Re}\langle y, e\rangle|^{p}}{\|y\|^{p}}\right)^{1 / p}\right| \leq\left(1-\frac{|\operatorname{Re}\langle x, y\rangle|^{p}}{\|x\|^{p}\|y\|^{p}}\right)^{1 / p}
$$


for any $x, y \neq 0$ and $e \in H$ with $\|e\|=1$.

If we take the power $p$ in (2.3) and (2.4) and multiply with $\|x\|^{p}\|y\|^{p}>0$, then we get the desired results (2.1) and (2.2).

The following similar result can be stated as well:

Theorem 2. Let $x, y, e \in H$ with $\|e\|=1$. Then we have the following refinement of Schwarz inequality

$$
\|x\|^{2}\|y\|^{2}-|\langle x, y\rangle|^{2} \geq\left(\operatorname{det}\left[\begin{array}{cc}
|\langle x, e\rangle| & \left(\|x\|^{2}-|\langle x, e\rangle|^{2}\right)^{1 / 2} \\
|\langle y, e\rangle| & \left(\|y\|^{2}-|\langle y, e\rangle|^{2}\right)^{1 / 2}
\end{array}\right]\right)^{2} .
$$

Proof. We have by Schwarz's inequality that

$$
|\langle x-\alpha e, y-\bar{\beta} e\rangle|^{2} \leq\|x-\alpha e\|^{2}\|y-\bar{\beta} e\|^{2}
$$

for any $x, y, e \in H$ and $\alpha, \beta \in \mathbb{C}$.

Since $\|e\|=1$, then

$$
\begin{gathered}
\langle x-\alpha e, y-\bar{\beta} e\rangle=\langle x, y\rangle-\alpha\langle e, y\rangle-\beta\langle x, e\rangle+\alpha \beta, \\
\|x-\alpha e\|^{2}=\|x\|^{2}-\left(2 \operatorname{Re}[\bar{\alpha}\langle x, e\rangle]-|\alpha|^{2}\right)
\end{gathered}
$$

and

$$
\|y-\bar{\beta} e\|^{2}=\|y\|^{2}-\left(2 \operatorname{Re}[\beta\langle y, e\rangle]-|\beta|^{2}\right) .
$$

This implies that

$$
\begin{aligned}
& \|x-\alpha e\|^{2}\|y-\bar{\beta} e\|^{2} \\
& =\left[\|x\|^{2}-\left(2 \operatorname{Re}[\bar{\alpha}\langle x, e\rangle]-|\alpha|^{2}\right)\right]\left[\|y\|^{2}-\left(2 \operatorname{Re}[\beta\langle x, e\rangle]-|\beta|^{2}\right)\right] \\
& =\|x\|^{2}\|y\|^{2}-\|y\|^{2}\left(2 \operatorname{Re}[\bar{\alpha}\langle x, e\rangle]-|\alpha|^{2}\right)-\|x\|^{2}\left(2 \operatorname{Re}[\beta\langle y, e\rangle]-|\beta|^{2}\right) \\
& +\left(2 \operatorname{Re}[\bar{\alpha}\langle x, e\rangle]-|\alpha|^{2}\right)\left(2 \operatorname{Re}[\beta\langle y, e\rangle]-|\beta|^{2}\right) \\
& =\|x\|^{2}\|y\|^{2}-\left(\|y\|^{2}-|\langle y, e\rangle|^{2}\right)\left(2 \operatorname{Re}[\bar{\alpha}\langle x, e\rangle]-|\alpha|^{2}\right) \\
& -\left(\|x\|^{2}-|\langle x, e\rangle|^{2}\right)\left(2 \operatorname{Re}[\beta\langle y, e\rangle]-|\beta|^{2}\right) \\
& -|\langle y, e\rangle|^{2}\left(2 \operatorname{Re}[\bar{\alpha}\langle x, e\rangle]-|\alpha|^{2}\right)-|\langle x, e\rangle|^{2}\left(2 \operatorname{Re}[\beta\langle y, e\rangle]-|\beta|^{2}\right) \\
& +\left(2 \operatorname{Re}[\bar{\alpha}\langle x, e\rangle]-|\alpha|^{2}\right)\left(2 \operatorname{Re}[\beta\langle y, e\rangle]-|\beta|^{2}\right) \\
& +|\langle y, e\rangle|^{2}|\langle x, e\rangle|^{2}-|\langle y, e\rangle|^{2}|\langle x, e\rangle|^{2} \\
& =\|x\|^{2}\|y\|^{2}-\left(\|y\|^{2}-|\langle y, e\rangle|^{2}\right)\left(2 \operatorname{Re}[\bar{\alpha}\langle x, e\rangle]-|\alpha|^{2}\right) \\
& -\left(\|x\|^{2}-|\langle x, e\rangle|^{2}\right)\left(2 \operatorname{Re}[\beta\langle y, e\rangle]-|\beta|^{2}\right) \\
& +\left[|\langle x, e\rangle|^{2}-\left(2 \operatorname{Re}[\bar{\alpha}\langle x, e\rangle]-|\alpha|^{2}\right)\right]\left[|\langle y, e\rangle|^{2}-\left(2 \operatorname{Re}[\beta\langle y, e\rangle]-|\beta|^{2}\right)\right] \\
& -|\langle y, e\rangle|^{2}|\langle x, e\rangle|^{2} .
\end{aligned}
$$


Observe that

$$
|\langle x, e\rangle|^{2}-\left(2 \operatorname{Re}[\bar{\alpha}\langle x, e\rangle]-|\alpha|^{2}\right)=|\langle x, e\rangle-\alpha|^{2}
$$

and

$$
|\langle y, e\rangle|^{2}-\left(2 \operatorname{Re}[\beta\langle y, e\rangle]-|\beta|^{2}\right)=|\langle y, e\rangle-\bar{\beta}|^{2} .
$$

Therefore, by (2.8) we get

$$
\begin{aligned}
& \|x-\alpha e\|^{2}\|y-\bar{\beta} e\|^{2} \\
& =\|x\|^{2}\|y\|^{2}-\left(\|y\|^{2}-|\langle y, e\rangle|^{2}\right)\left(2 \operatorname{Re}[\bar{\alpha}\langle x, e\rangle]-|\alpha|^{2}\right) \\
& -\left(\|x\|^{2}-|\langle x, e\rangle|^{2}\right)\left(2 \operatorname{Re}[\beta\langle y, e\rangle]-|\beta|^{2}\right) \\
& +|\langle x, e\rangle-\alpha|^{2}|\langle y, e\rangle-\bar{\beta}|^{2}-|\langle y, e\rangle|^{2}|\langle x, e\rangle|^{2} .
\end{aligned}
$$

Let $\alpha \in \mathbb{C}$ with $\alpha \neq\langle x, e\rangle$ and put

$$
\beta:=\frac{\alpha \overline{\langle y, e\rangle}}{\alpha-\langle x, e\rangle}
$$

Then

$$
\begin{aligned}
|\langle x, e\rangle-\alpha|^{2}|\langle y, e\rangle-\bar{\beta}|^{2} & =|\langle x, e\rangle-\alpha|^{2}\left|\langle y, e\rangle-\overline{\left(\frac{\alpha \overline{\langle y, e\rangle}}{\alpha-\langle x, e\rangle}\right)}\right|^{2} \\
& =|\langle x, e\rangle-\alpha|^{2}\left|\langle y, e\rangle-\frac{\bar{\alpha}\langle y, e\rangle}{\bar{\alpha}-\overline{\langle x, e\rangle}}\right|^{2} \\
& =|\langle y, e\rangle|^{2}|\langle x, e\rangle-\alpha|^{2}\left|\frac{\overline{\langle x, e\rangle}}{\overline{\bar{\alpha}-\overline{\langle x, e\rangle}}}\right|^{2} \\
& =|\langle y, e\rangle|^{2}|\langle x, e\rangle|^{2}
\end{aligned}
$$

and

$$
\alpha \beta=\alpha\langle e, y\rangle+\beta\langle x, e\rangle .
$$

For these choices of $\alpha$ and $\beta$ we have by (2.6)-(2.9) that

$$
\begin{aligned}
|\langle x, y\rangle|^{2} & \leq\|x\|^{2}\|y\|^{2}-\left(\|y\|^{2}-|\langle y, e\rangle|^{2}\right)\left(2 \operatorname{Re}[\bar{\alpha}\langle x, e\rangle]-|\alpha|^{2}\right) \\
& -\left(\|x\|^{2}-|\langle x, e\rangle|^{2}\right)\left(2 \operatorname{Re}[\beta\langle y, e\rangle]-|\beta|^{2}\right) .
\end{aligned}
$$

By (2.10) we also have

$$
2 \operatorname{Re}[\beta\langle y, e\rangle]-|\beta|^{2}=|\langle y, e\rangle|^{2}\left[2 \operatorname{Re}\left[\frac{\alpha}{\alpha-\langle x, e\rangle}\right]-\left|\frac{\alpha}{\alpha-\langle x, e\rangle}\right|^{2}\right] .
$$

Take

$$
\alpha=\langle x, e\rangle+t \text { with } t \in \mathbb{R}, t \neq 0 \text {. }
$$


Then by (2.12) we have

$$
\begin{aligned}
B(x, y, e, t) & :=\left(\|y\|^{2}-|\langle y, e\rangle|^{2}\right)\left(2 \operatorname{Re}[\bar{\alpha}\langle x, e\rangle]-|\alpha|^{2}\right) \\
& +\left(\|x\|^{2}-|\langle x, e\rangle|^{2}\right)\left(2 \operatorname{Re}[\beta\langle y, e\rangle]-|\beta|^{2}\right) \\
& =\left(\|y\|^{2}-|\langle y, e\rangle|^{2}\right)\left(2 \operatorname{Re}[(\overline{\langle x, e\rangle+t})\langle x, e\rangle]-|\langle x, e\rangle+t|^{2}\right) \\
& +\left(\|x\|^{2}-|\langle x, e\rangle|^{2}\right)|\langle y, e\rangle|^{2}\left[2 \operatorname{Re}\left[\frac{\langle x, e\rangle+t}{t}\right]-\left|\frac{\langle x, e\rangle+t}{t}\right|^{2}\right] .
\end{aligned}
$$

Since

$$
\begin{aligned}
& 2 \operatorname{Re}[(\overline{\langle x, e\rangle+t})\langle x, e\rangle]-|\langle x, e\rangle+t|^{2} \\
& =2 \operatorname{Re}\left[|\langle x, e\rangle|^{2}+t\langle x, e\rangle\right]-|\langle x, e\rangle|^{2}-2 t \operatorname{Re}\langle x, e\rangle-t^{2} \\
& =|\langle x, e\rangle|^{2}-t^{2}
\end{aligned}
$$

and

$$
\begin{aligned}
& 2 \operatorname{Re}\left[\frac{\langle x, e\rangle+t}{t}\right]-\left|\frac{\langle x, e\rangle+t}{t}\right|^{2} \\
& =2 \operatorname{Re}\left[\frac{\langle x, e\rangle}{t}+1\right]-\left|\frac{\langle x, e\rangle}{t}+1\right|^{2} \\
& =\frac{2 \operatorname{Re}\langle x, e\rangle}{t}+2-\frac{|\langle x, e\rangle|^{2}}{t^{2}}-\frac{2 \operatorname{Re}\langle x, e\rangle}{t}-1=1-\frac{|\langle x, e\rangle|^{2}}{t^{2}},
\end{aligned}
$$

then we get

$$
\begin{aligned}
B(x, y, e, t) & =\left(\|y\|^{2}-|\langle y, e\rangle|^{2}\right)\left(|\langle x, e\rangle|^{2}-t^{2}\right) \\
& +\left(\|x\|^{2}-|\langle x, e\rangle|^{2}\right)|\langle y, e\rangle|^{2}\left(1-\frac{|\langle x, e\rangle|^{2}}{t^{2}}\right) \\
& =\left(\|y\|^{2}-|\langle y, e\rangle|^{2}\right)\left(|\langle x, e\rangle|^{2}-t^{2}\right) \\
& -\left(\|x\|^{2}-|\langle x, e\rangle|^{2}\right)|\langle y, e\rangle|^{2}\left(\frac{|\langle x, e\rangle|^{2}-t^{2}}{t^{2}}\right) \\
& =\left(|\langle x, e\rangle|^{2}-t^{2}\right)\left[\|y\|^{2}-|\langle y, e\rangle|^{2}-\frac{\left(\|x\|^{2}-|\langle x, e\rangle|^{2}\right)|\langle y, e\rangle|^{2}}{t^{2}}\right]
\end{aligned}
$$

for $t \in \mathbb{R}, t \neq 0$.

Assume that $\langle x, e\rangle,\langle y, e\rangle \neq 0$ and $\|x\| \neq|\langle x, e\rangle|,\|y\| \neq|\langle y, e\rangle|$.

If we take $t=t_{0} \neq 0$ with

$$
t_{0}^{2}=|\langle x, e\rangle\langle y, e\rangle| \sqrt{\frac{\|x\|^{2}-|\langle x, e\rangle|^{2}}{\|y\|^{2}-|\langle y, e\rangle|^{2}}}
$$


then we get

$$
\begin{aligned}
B & \left(x, y, e, t_{0}\right) \\
= & \left(|\langle x, e\rangle|^{2}-|\langle x, e\rangle\langle y, e\rangle| \sqrt{\frac{\|x\|^{2}-|\langle x, e\rangle|^{2}}{\|y\|^{2}-|\langle y, e\rangle|^{2}}}\right) \\
\times & {\left[\|y\|^{2}-|\langle y, e\rangle|^{2}-\frac{\left(\|x\|^{2}-|\langle x, e\rangle|^{2}\right)|\langle y, e\rangle|^{2}}{|\langle x, e\rangle\langle y, e\rangle| \sqrt{\frac{\|x\|^{2}-|\langle x, e\rangle|^{2}}{\|y\|^{2}-|\langle y, e\rangle|^{2}}}}\right] } \\
= & \frac{|\langle x, e\rangle|}{\sqrt{\|y\|^{2}-|\langle y, e\rangle|^{2}}}\left(|\langle x, e\rangle| \sqrt{\|y\|^{2}-|\langle y, e\rangle|^{2}}-|\langle y, e\rangle| \sqrt{\|x\|^{2}-|\langle x, e\rangle|^{2}}\right) \\
\times & {\left[\frac{|\langle x, e\rangle| \sqrt{\|y\|^{2}-|\langle y, e\rangle|^{2}}-\sqrt{\|x\|^{2}-|\langle x, e\rangle|^{2}}|\langle y, e\rangle|}{|\langle x, e\rangle|} \sqrt{\|y\|^{2}-|\langle y, e\rangle|^{2}}\right.} \\
= & \left(|\langle x, e\rangle| \sqrt{\|y\|^{2}-|\langle y, e\rangle|^{2}}-|\langle y, e\rangle| \sqrt{\|x\|^{2}-|\langle x, e\rangle|^{2}}\right)^{2} .
\end{aligned}
$$

By using the inequality (2.11) we then have

$$
\begin{aligned}
|\langle x, y\rangle|^{2} & \leq\|x\|^{2}\|y\|^{2}-B\left(x, y, e, t_{0}\right) \\
& =\|x\|^{2}\|y\|^{2}-\left(|\langle x, e\rangle| \sqrt{\|y\|^{2}-|\langle y, e\rangle|^{2}}-|\langle y, e\rangle| \sqrt{\|x\|^{2}-|\langle x, e\rangle|^{2}}\right)^{2}
\end{aligned}
$$

which proves the desired result (2.5).

Now, if $\langle x, e\rangle=0$ i.e. $x \perp e$, then (2.5) becomes

$$
|\langle y, e\rangle|^{2}\|x\|^{2}+|\langle x, y\rangle|^{2} \leq\|x\|^{2}\|y\|^{2}
$$

which is trivial for $x=0$ and becomes the Bessel's inequality

$$
|\langle y, e\rangle|^{2}+\left|\left\langle y, \frac{x}{\|x\|}\right\rangle\right|^{2} \leq\|y\|^{2}
$$

for the orthonormal family $\left\{e, \frac{x}{\|x\|}\right\}$.

A similar argument applies for $\langle y, e\rangle=0$.

Also, if $\|x\|^{2}=|\langle x, e\rangle|^{2}$ then by the equality case in Schwarz inequality for the vectors $x$ and $e$ we get that there exists a constant $\gamma$ such that $x=\gamma e$. In this situation (2.5) becomes an equality.

A similar argument applies if $\|y\|^{2}=|\langle y, e\rangle|^{2}$.

Remark 1. If $(H,\langle\cdot, \cdot\rangle)$ is a complex inner product space, then $\left(H,\langle\cdot, \cdot\rangle_{r}\right)$ with

$$
\langle x, y\rangle_{r}:=\operatorname{Re}\langle x, y\rangle
$$


is a real inner product space and $\langle x, x\rangle^{1 / 2}=\langle x, x\rangle_{r}^{1 / 2}=\|x\|$ for $x \in H$. Therefore by (2.5) for $\langle\cdot, \cdot\rangle_{r}$ we get

$$
\begin{aligned}
& \|x\|^{2}\|y\|^{2}-|\operatorname{Re}\langle x, y\rangle|^{2} \\
& \geq\left(\operatorname{det}\left[\begin{array}{cc}
|\operatorname{Re}\langle x, e\rangle| & \left(\|x\|^{2}-|\operatorname{Re}\langle x, e\rangle|^{2}\right)^{1 / 2} \\
|\operatorname{Re}\langle y, e\rangle| & \left(\|y\|^{2}-|\operatorname{Re}\langle y, e\rangle|^{2}\right)^{1 / 2}
\end{array}\right]\right)^{2}
\end{aligned}
$$

for any $x, y, e \in H$ with $\|e\|=1$.

\section{An Application for $n$-Tuples of Complex Numbers}

Let $x=\left(x_{1}, \ldots, x_{n}\right), y=\left(y_{1}, \ldots, y_{n}\right), e=\left(e_{1}, \ldots, e_{n}\right) \in \mathbb{C}^{n}$ with $\sum_{k=1}^{n}\left|e_{k}\right|^{2}=$

1. Then by writing the above inequalities (2.1) and (2.5) for the inner product $\langle x, y\rangle:=\sum_{k=1}^{n} x_{k} \bar{y}_{k}$ we have for $p \geq 2$, that

$$
\begin{aligned}
& \left(\sum_{k=1}^{n}\left|x_{k}\right|^{2}\right)^{p / 2}\left(\sum_{k=1}^{n}\left|y_{k}\right|^{2}\right)^{p / 2}-\left|\sum_{k=1}^{n} x_{k} \bar{y}_{k}\right|^{p} \\
& \left.\geq \operatorname{det}\left[\begin{array}{cc}
\left(\sum_{k=1}^{n}\left|x_{k}\right|^{2}\right)^{1 / 2} & \left(\left(\sum_{k=1}^{n}\left|x_{k}\right|^{2}\right)^{p / 2}-\left|\sum_{k=1}^{n} x_{k} \bar{e}_{k}\right|^{p}\right)^{1 / p} \\
\left(\sum_{k=1}^{n}\left|y_{k}\right|^{2}\right)^{1 / 2} & \left(\left(\sum_{k=1}^{n}\left|y_{k}\right|^{2}\right)^{p / 2}-\left|\sum_{k=1}^{n} y_{k} \bar{e}_{k}\right|^{p}\right)^{1 / p}
\end{array}\right]\right)^{p},
\end{aligned}
$$

and

$$
\begin{aligned}
& \sum_{k=1}^{n}\left|x_{k}\right|^{2} \sum_{k=1}^{n}\left|y_{k}\right|^{2}-\left|\sum_{k=1}^{n} x_{k} \bar{y}_{k}\right|^{2} \\
& \left.\geq \operatorname{det}\left[\begin{array}{cc}
\left|\sum_{k=1}^{n} x_{k} \bar{e}_{k}\right| & \left(\sum_{k=1}^{n}\left|x_{k}\right|^{2}-\left|\sum_{k=1}^{n} x_{k} \bar{e}_{k}\right|^{2}\right)^{1 / 2} \\
\left|\sum_{k=1}^{n} y_{k} \bar{e}_{k}\right| & \left(\sum_{k=1}^{n}\left|y_{k}\right|^{2}-\left|\sum_{k=1}^{n} y_{k} \bar{e}_{k}\right|^{2}\right)^{1 / 2}
\end{array}\right]\right)^{2} .
\end{aligned}
$$

If we take $e_{m}=1$ for $m \in\{1, \ldots, n\}$ and $e_{k}=0$ for any $k \in\{1, \ldots, n\}, k \neq m$, then $\sum_{k=1}^{n}\left|e_{k}\right|^{2}=1$ and by (3.1) and (3.2) we get

$$
\begin{aligned}
& \text { (3.3) }\left(\sum_{k=1}^{n}\left|x_{k}\right|^{2}\right)^{p / 2}\left(\sum_{k=1}^{n}\left|y_{k}\right|^{2}\right)^{p / 2}-\left|\sum_{k=1}^{n} x_{k} \bar{y}_{k}\right|^{p} \\
& \geq \max _{m \in\{1, \ldots, n\}}\left(\operatorname{det}\left[\begin{array}{cc}
\left(\sum_{k=1}^{n}\left|x_{k}\right|^{2}\right)^{1 / 2} & \left(\left(\sum_{k=1}^{n}\left|x_{k}\right|^{2}\right)^{p / 2}-\left|x_{m}\right|^{p}\right)^{1 / p} \\
\left(\sum_{k=1}^{n}\left|y_{k}\right|^{2}\right)^{1 / 2} & \left(\left(\sum_{k=1}^{n}\left|y_{k}\right|^{2}\right)^{p / 2}-\left|y_{m}\right|^{p}\right)^{1 / p}
\end{array}\right]\right)^{p},
\end{aligned}
$$


and

$$
\begin{aligned}
& \sum_{k=1}^{n}\left|x_{k}\right|^{2} \sum_{k=1}^{n}\left|y_{k}\right|^{2}-\left|\sum_{k=1}^{n} x_{k} \bar{y}_{k}\right|^{2} \\
& \geq \max _{m \in\{1, \ldots, n\}}\left(\operatorname{det}\left[\begin{array}{cc}
\left|x_{m}\right| & \left(\sum_{1 \leq k \neq m \leq n}\left|x_{k}\right|^{2}\right)^{1 / 2} \\
\left|y_{m}\right| & \left(\sum_{1 \leq k \neq m \leq n}\left|y_{k}\right|^{2}\right)^{1 / 2}
\end{array}\right]\right)^{2} .
\end{aligned}
$$

For $p=2$ we get from (3.3) the simpler inequality

$$
\begin{aligned}
& \sum_{k=1}^{n}\left|x_{k}\right|^{2} \sum_{k=1}^{n}\left|y_{k}\right|^{2}-\left|\sum_{k=1}^{n} x_{k} \bar{y}_{k}\right|^{2} \\
& \quad \geq \max _{m \in\{1, \ldots, n\}}\left(\operatorname{det}\left[\begin{array}{cc}
\left(\sum_{k=1}^{n}\left|x_{k}\right|^{2}\right)^{1 / 2} & \left(\sum_{1 \leq k \neq m \leq n}\left|x_{k}\right|^{2}\right)^{1 / 2} \\
\left(\sum_{k=1}^{n}\left|y_{k}\right|^{2}\right)^{1 / 2} & \left(\sum_{1 \leq k \neq m \leq n}\left|y_{k}\right|^{2}\right)^{1 / 2}
\end{array}\right]\right)^{2} .
\end{aligned}
$$

If we take $e_{k}=\frac{1}{\sqrt{n}}$ for $k \in\{1, \ldots, n\}$, then $\sum_{k=1}^{n}\left|e_{k}\right|^{2}=1$ and by (3.1) and (3.2) we get

$$
\begin{aligned}
& \text { (3.6) } \quad\left(\sum_{k=1}^{n}\left|x_{k}\right|^{2}\right)^{p / 2}\left(\sum_{k=1}^{n}\left|y_{k}\right|^{2}\right)^{p / 2}-\left|\sum_{k=1}^{n} x_{k} \bar{y}_{k}\right|^{p} \\
& \left.\geq \operatorname{det}\left[\begin{array}{cc}
\left(\frac{1}{n} \sum_{k=1}^{n}\left|x_{k}\right|^{2}\right)^{1 / 2} & \left(\left(\frac{1}{n} \sum_{k=1}^{n}\left|x_{k}\right|^{2}\right)^{p / 2}-\left|\frac{1}{n} \sum_{k=1}^{n} x_{k}\right|^{p}\right)^{1 / p} \\
\left(\frac{1}{n} \sum_{k=1}^{n}\left|y_{k}\right|^{2}\right)^{1 / 2} & \left(\left(\frac{1}{n} \sum_{k=1}^{n}\left|y_{k}\right|^{2}\right)^{p / 2}-\left|\frac{1}{n} \sum_{k=1}^{n} y_{k}\right|^{p}\right)^{1 / p}
\end{array}\right]\right)
\end{aligned}
$$

and

$$
\begin{aligned}
& \sum_{k=1}^{n}\left|x_{k}\right|^{2} \sum_{k=1}^{n}\left|y_{k}\right|^{2}-\left|\sum_{k=1}^{n} x_{k} \bar{y}_{k}\right|^{2} \\
& \geq n^{2}\left(\operatorname{det}\left[\begin{array}{cc}
\left|\frac{1}{n} \sum_{k=1}^{n} x_{k}\right| & \left(\frac{1}{n} \sum_{k=1}^{n}\left|x_{k}\right|^{2}-\left|\frac{1}{n} \sum_{k=1}^{n} x_{k}\right|^{2}\right)^{1 / 2} \\
\left|\frac{1}{n} \sum_{k=1}^{n} y_{k}\right| & \left(\frac{1}{n} \sum_{k=1}^{n}\left|y_{k}\right|^{2}-\left|\frac{1}{n} \sum_{k=1}^{n} y_{k}\right|^{2}\right)^{1 / 2}
\end{array}\right]\right)^{2} .
\end{aligned}
$$

The inequality (3.7) has been obtained recently for real numbers by S. G. Walker in [9], where some interesting applications for the celebrated Cramer-Rao inequality are provided as well.

\section{REFERENCES}

[1] S. S. Dragomir, Some refinements of Schwarz inequality, Simpozionul de Matematici şi Aplicaţii, Timişoara, Romania, 1-2 Noiembrie 1985, 13-16. ZBL 0594.46018. 
[2] S. S. Dragomir and I. Sándor, Some inequalities in pre-Hilbertian spaces. Studia Univ. BabeşBolyai Math. 32 (1987), no. 1, 71-78.

[3] S. S. Dragomir, Advances in Inequalities of the Schwarz, Grüss and Bessel Type in Inner Product Spaces. Nova Science Publishers, Inc., Hauppauge, NY, 2005. viii+249 pp. ISBN: 1-59454-202-3.

[4] S. S. Dragomir, Advances in Inequalities of the Schwarz, Triangle and Heisenberg Type in Inner Product Spaces. Nova Science Publishers, Inc., New York, 2007. xii+243 pp. ISBN: 978-1-59454-903-8; 1-59454-903-6.

[5] S. S. Dragomir, Buzano's inequality holds for any projection, Bull. Aust. Math. Soc. 93 (2016), 504-510.

[6] K. E. Gustafson and D. K. M. Rao, Numerical Range, Springer-Verlag, New York, Inc., 1997.

[7] M. K. KreĬn, Angular localization of the spectrum of a multiplicative integral in a Hilbert space, Funct. Anal. Appl. 3 (1969), 89-90.

[8] M. Lin, Remarks on Kreĭn's inequality, The Math. Intelligencer, 34 (2012), No.1, 3-4.

[9] S. G. Walker, A self-improvement to the Cauchy-Schwarz inequality, Statistics and Probability Letters 122 (2017), 86-89.

[10] B. Wang, F. Zhang, A trace inequality for unitary matrices, Amer. Math. Monthly 101 (1994), 453-455.

[11] F. Zhang, Matrix Theory: Basic Results and Techniques, Springer-Verlag, New York, 2011.

${ }^{1}$ Mathematics, College of Engineering \& Science, Victoria University, PO Box 14428 , Melbourne City, MC 8001, Australia.

E-mail address: sever.dragomir@vu.edu.au

$U R L:$ http://rgmia.org/dragomir

${ }^{2}$ DST-NRF Centre of Excellence, in the Mathematical and Statistical Sciences, School of Computer Science \& Applied Mathematics, University of the Witwatersrand, Private Bag 3, Johannesburg 2050, South Africa 